\title{
Normalized Fluorescence Intensity Unit
}

National Cancer Institute

\section{Source}

National Cancer Institute. Normalized Fluorescence Intensity Unit. NCI Thesaurus. Code C154680.

A relative fluorescence intensity unit that is adjusted to a reference standard. 